\title{
A Systems-level Analysis of Waste Heat Recovery Opportunities from Natural Gas Compressor Stations in the U.S.
}

\author{
Sakineh Tavakkoli, Omkar R. Lokare, Radisav D. Vidic, and Vikas Khanna*
}

Department of Civil and Environmental Engineering, University of Pittsburgh, 3700 O'Hara

Street, 742 Benedum Hall, Pittsburgh, Pennsylvania 15261, United States

*Address correspondence to khannav@pitt.edu

KEYWORDS: natural gas, compressor stations, thermodynamic analysis, uncertainty assessment, greenhouse gas emissions

\section{Overview of the Study}

The goal of our study is to evaluate the quantity and quality of available waste heat at natural compressor stations in the United States using thermodynamic analysis and engineering calculations.

\section{Compressor stations mechanical drive type}

Table S1 Probability distribution for having an internal combustion engine within different installed capacity ranges stated as horsepower (HP)

\begin{tabular}{ccc}
\hline $\begin{array}{c}\text { Lower capacity range } \\
\text { (HP) }\end{array}$ & $\begin{array}{c}\text { Upper capacity } \\
\text { range (HP) }\end{array}$ & $\begin{array}{c}\text { Probability of being } \\
\text { Internal Combustion }\end{array}$ \\
\hline 42 & 800 & 1.00 \\
800 & 1558 & 0.91 \\
1558 & 2316 & 0.98 \\
2316 & 3075 & 1.00 \\
3075 & 3833 & 0.83 \\
3833 & 4591 & 0.75 \\
4591 & 5349 & 0.62 \\
5349 & 6107 & 0.71 \\
6107 & 6865 & 0.63 \\
6865 & 7624 & 0.40 \\
7624 & 8382 & 0.67 \\
8382 & 9140 & 0.13 \\
9140 & 9898 & 0.00 \\
9898 & 10656 & 0.29 \\
10656 & 11414 & 0.25 \\
11414 & 12173 & 0.33 \\
12173 & 12931 & 0.00 \\
\hline
\end{tabular}




\begin{tabular}{|c|c|c|}
\hline 12931 & 13689 & 0.00 \\
\hline 13689 & 14447 & 0.00 \\
\hline 14447 & 15205 & 0.00 \\
\hline 15205 & 15963 & 0.00 \\
\hline 15963 & 16722 & 0.00 \\
\hline 16722 & 17480 & 0.00 \\
\hline 17480 & 18238 & 0.00 \\
\hline 18238 & 18996 & 0.00 \\
\hline 18996 & 19754 & 0.00 \\
\hline 19754 & 20512 & 0.00 \\
\hline 20512 & 21270 & 0.00 \\
\hline 21270 & 22029 & 0.00 \\
\hline 22029 & 22787 & 0.00 \\
\hline 22787 & 23545 & 0.00 \\
\hline 23545 & 24303 & 0.00 \\
\hline 24303 & 25061 & 0.00 \\
\hline 25061 & 25819 & 0.00 \\
\hline 25819 & 26578 & 0.00 \\
\hline 26578 & 27336 & 0.00 \\
\hline 27336 & 28094 & 0.00 \\
\hline 28094 & 28852 & 0.00 \\
\hline 28852 & 29610 & 0.00 \\
\hline 29610 & 30368 & 0.00 \\
\hline 30368 & 31127 & 0.00 \\
\hline 31127 & 31885 & 0.00 \\
\hline 31885 & 32643 & 0.00 \\
\hline 32643 & 33401 & 0.00 \\
\hline 33401 & 34159 & 0.00 \\
\hline 34159 & 34917 & 0.00 \\
\hline 34917 & 35676 & 0.00 \\
\hline 35676 & 36434 & 0.00 \\
\hline 36434 & 37192 & 0.00 \\
\hline 37192 & 37950 & 0.00 \\
\hline
\end{tabular}




\section{Thermodynamic analysis for waste heat estimation}

\section{Gas turbine CS}

Figure S1 shows a process schematic of a typical gas turbine NG CS.

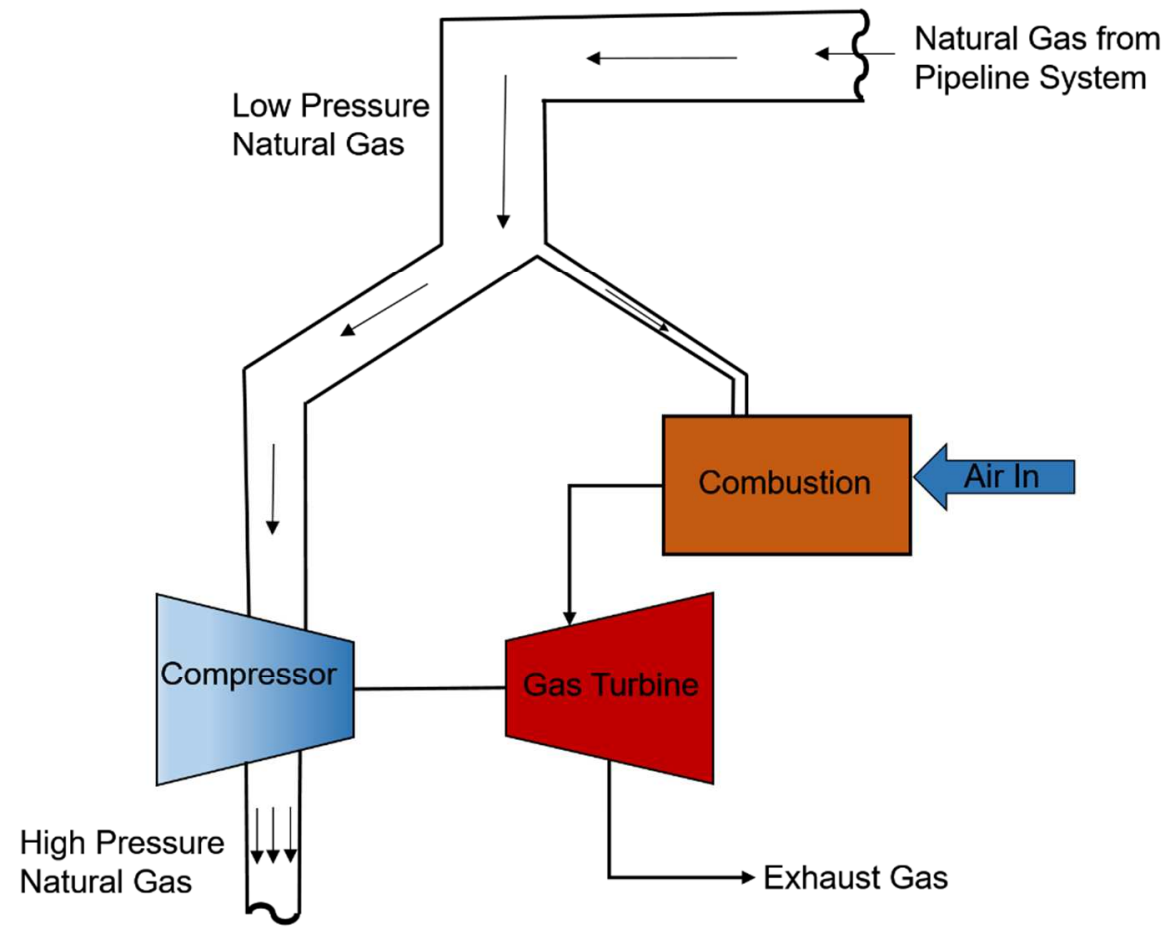

Figure S1 Simplified flow diagram of compressing NG and generating waste heat at gas turbine NG CS. ${ }^{1}$

\section{Adiabatic flame temperature calculation}

In order to calculate the adiabatic flame temperature, ${ }^{2}$ we define the initial condition of 1 atm pressure, $298^{\circ} \mathrm{K}$ initial temperature and a 3.76 molar ratio for nitrogen to oxygen in the air. We also need to calculate fuel-air equivalence ratio $(\varnothing)$ which is defined as the ratio of actual fuel-toair ratio $\left(\left(\frac{F u e l}{\text { air }}\right)_{a c}\right)$ to stoichiometry fuel-to-air ratio $\left(\left(\frac{F u e l}{\text { air }}\right)_{s t}\right)$ for a given mixture. ${ }^{3}$ The actual combustion reaction with $100 \%$ excess air is written as eq 1 and the stoichiometric reaction is shown as eq 2 . The equivalence ratio is then calculated as shown in eq 3 .

$$
\begin{aligned}
& \mathrm{CH}_{4}+2 * 2\left(\mathrm{O}_{2}+3.67 \mathrm{~N}_{2}\right) \rightarrow \mathrm{CO}_{2}+2 \mathrm{H}_{2} \mathrm{O}+2 \mathrm{O}_{2}+14.68 \mathrm{~N}_{2} \\
& \mathrm{CH}_{4}+2\left(\mathrm{O}_{2}+3.67 \mathrm{~N}_{2}\right) \rightarrow \mathrm{CO}_{2}+2 \mathrm{H}_{2} \mathrm{O}+7.34 \mathrm{~N}_{2} \\
& \varnothing=\frac{\left(\frac{\text { Fuel }}{\text { air }}\right)_{a c}}{\left(\frac{\text { Fuel }}{\text { air }}\right)_{s t}}=\frac{\left(\frac{12+4}{2 * 2 * 32}\right)}{\left(\frac{12+4}{2 * 32}\right)}=0.5
\end{aligned}
$$


We use Cantera online software toolkit ${ }^{4}$ to calculate the adiabatic flame temperature and the resulting temperature is $1478^{\circ} \mathrm{K}$ for combustion of methane with $100 \%$ excess air.

Flue gas composition and enthalpy calculations

The fuel composition for natural gas is shown in Table S2. We assume complete combustion with $10 \%$ excess air where air is composed of $21 \%$ oxygen and $79 \%$ Nitrogen.

Table S2 Assumed fuel composition (volumetric percentage)

\begin{tabular}{|c|c|}
\hline \multicolumn{2}{|c|}{ Natural Gas } \\
\hline Methane $\left(\mathrm{CH}_{4}\right)$ & $93.27 \%$ \\
\hline Ethane $\left(\mathrm{C}_{2} \mathrm{H}_{6}\right)$ & $3.79 \%$ \\
\hline Propane $\left(\mathrm{C}_{3} \mathrm{H}_{8}\right)$ & $0.57 \%$ \\
\hline Butane $\left(\mathrm{C}_{4} \mathrm{H}_{10}\right)$ & $0.29 \%$ \\
\hline Nitrogen & $1.19 \%$ \\
\hline Water & $0.00 \%$ \\
\hline Carbon Dioxide & $0.79 \%$ \\
\hline
\end{tabular}

Methane is the primary constituent of the NG and the stoichiometric equation for combustion of methane with $10 \%$ excess air is shown eq 1 .

$$
\mathrm{CH}_{4}+1.1 * 2\left(\mathrm{O}_{2}+3.67 \mathrm{~N}_{2}\right) \rightarrow \mathrm{CO}_{2}+2 \mathrm{H}_{2} \mathrm{O}+0.2 \mathrm{O}_{2}+8.272 \mathrm{~N}_{2}
$$

The combustion equation is written for each species in the fuel and the mole fraction of combustion products in the exhaust gas is determined as shown in Table S3. These values are used to calculate the enthalpy for each constituent of exhaust gas.

Table S3 Exhaust gas composition ${ }^{5}$

\begin{tabular}{|l|l|}
\hline Flue Gas Species & Natural Gas \\
\hline $\mathrm{CO}_{2}$ & $9.7 \%$ \\
\hline $\mathrm{H}_{2} \mathrm{O}$ & $18.7 \%$ \\
\hline $\mathrm{N}_{2}$ & $71.6 \%$ \\
\hline
\end{tabular}

Enthalpy values for each species in gas turbine inlet and outlet stream are calculated based on the temperature as following.

(a) $T_{\text {in }}=1400^{\circ} \mathrm{K}$ :

$$
\begin{gathered}
h_{\mathrm{Co}_{2}}=65271 \mathrm{~kJ} / \mathrm{kmol} \\
h_{\mathrm{H}_{2} \mathrm{O}}=55351 \mathrm{~kJ} / \mathrm{kmol} \\
h_{\mathrm{N}_{2}}=43605 \mathrm{~kJ} / \mathrm{kmol}
\end{gathered}
$$

(a) $T_{\text {in }}=921^{\circ} \mathrm{K}$ :

$$
\begin{gathered}
h_{\mathrm{Co}_{2}}=38467 \mathrm{~kJ} / \mathrm{kmol} \\
h_{\mathrm{H}_{2} \mathrm{O}}=32629 \mathrm{~kJ} / \mathrm{kmol} \\
h_{\mathrm{N}_{2}}=27532 \mathrm{~kJ} / \mathrm{kmol}
\end{gathered}
$$


The mass flow rate of exhaust gas is calculated as shown in eq 5 . These calculations are carried out as an example for a compressor station with the capacity of $12000 \mathrm{HP}(32214236.6 \mathrm{~kJ} / \mathrm{hr})$ which is the average capacity of a compressor station in the U.S.

$32214236.6=\dot{m}(0.097(65217-38467)+0.187(55351-32629)+0.716(43606-$

27532))

$$
\dot{m}=1755.35 \mathrm{kmol} / \mathrm{hr}
$$

In order to estimate the heat contained in the exhaust stream, we assume that the exhaust stream will be cooled down to $60^{\circ} \mathrm{C}\left(333^{\circ} \mathrm{K}\right) .^{5,7}$ Available waste heat at this compressor station is calculated as shown in eq 6 .

(a) $T_{\text {in }}=921^{\circ} \mathrm{K}$ :

$$
\begin{gathered}
h_{\mathrm{Co}_{2}}=38467 \mathrm{~kJ} / \mathrm{kmol} \\
h_{\mathrm{H}_{2} \mathrm{O}}=32629 \mathrm{~kJ} / \mathrm{kmol} \\
h_{N_{2}}=27532 \mathrm{~kJ} / \mathrm{kmol}
\end{gathered}
$$

@ $T_{\text {in }}=333^{\circ} \mathrm{K}$ :

$$
\begin{aligned}
& h_{\mathrm{Co}_{2}}=10570 \mathrm{~kJ} / \mathrm{kmol} \\
& h_{\mathrm{H}_{2} \mathrm{O}}=10976 \mathrm{~kJ} / \mathrm{kmol} \\
& h_{\mathrm{N}_{2}}=9597 \mathrm{~kJ} / \mathrm{kmol}
\end{aligned}
$$

$q=\dot{m}\left(h_{\text {in }}-h_{\text {out }}\right)=1755.35 \frac{\mathrm{kmol}}{\mathrm{hr}} *(29545.834-9949.254) \frac{\mathrm{kJ}}{\mathrm{kmol}}=34398853.74 \frac{\mathrm{kJ}}{\mathrm{hr}}$

Table S4 List of all mathematical symbols and definition

\begin{tabular}{c|l} 
Symbol & Definition \\
\hline$\dot{W}_{\text {comp }}$ & Power of compressor station \\
$\dot{W}_{\text {turb }}$ & Power of gas turbine \\
$\dot{m}$ & Mass flow rate \\
$h_{\text {in }}$ & Inlet enthalpy \\
$h_{\text {out }}$ & Outlet enthalpy \\
$T_{\text {in }}$ & Inlet temperature \\
$T_{\text {out }}$ & Outlet temperature \\
$P_{\text {in }}$ & Inlet Pressure \\
$P_{\text {out }}$ & Outlet Pressure \\
$\eta$ & Gas turbine isentropic efficiency \\
$k$ & Ratio of specific heat \\
$h_{C o_{2}}$ & Enthalpy of Carbon Dioxide \\
$h_{\mathrm{H}_{2} \mathrm{O}}$ & Enthalpy of Steam \\
$h_{\mathrm{N}_{2}}$ & Enthalpy of Nitrogen Gas
\end{tabular}




\section{Reciprocating internal combustion (IC) engine CS}

Figure S2 shows the schematic of four principal states of a cycle in an IC engine. Each cycle consists of two processes (processes 1-2 and 3-4) in which there is work but no heat transfer and two processes (processes 2-3 and 4-1) in which there is heat transfer but no work. ${ }^{6}$

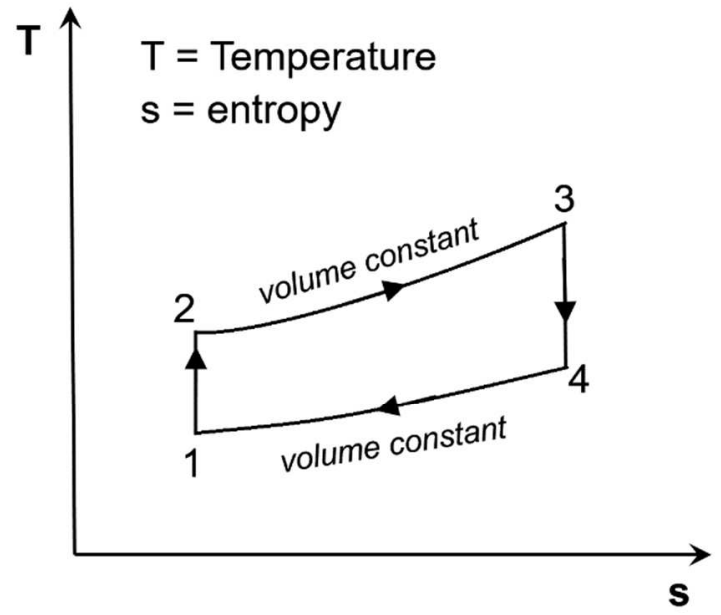

Figure S2 T-s diagram of four principal states of a cycle in an IC engine ${ }^{6}$

\section{State specific electricity mix for year 2013}

Table S5 Electricity generation mix by state ${ }^{8}$

\begin{tabular}{|c|c|c|c|}
\hline Alaska (AK) & $\%$ & Montana (MT) & $\%$ \\
\hline Coal & 9.61 & Coal & 53.74 \\
\hline Hydroelectric Conventional & 22.10 & Hydroelectric Conventional & 34.81 \\
\hline Natural Gas & 52.66 & Natural Gas & 2.22 \\
\hline Other Biomass & 0.80 & Other & 1.20 \\
\hline Petroleum & 12.60 & Other Gases & 0.00 \\
\hline Wind & 2.24 & Petroleum & 1.67 \\
\hline \multicolumn{2}{|l|}{ Alabama (AL) } & Wind & 6.34 \\
\hline Coal & 31.25 & Wood and Wood Derived Fuels & 0.02 \\
\hline Hydroelectric Conventional & 8.57 & \multicolumn{2}{|l|}{ North Carolina (NC) } \\
\hline Natural Gas & 30.94 & Coal & 37.38 \\
\hline Nuclear & 27.11 & Hydroelectric Conventional & 5.48 \\
\hline Other Biomass & 0.01 & Natural Gas & 22.22 \\
\hline Other Gases & 0.18 & Nuclear & 31.95 \\
\hline Petroleum & 0.05 & Other & 0.45 \\
\hline Wood and Wood Derived Fuels & 1.90 & Other Biomass & 0.33 \\
\hline \multicolumn{2}{|l|}{ Arkansas (AR) } & Petroleum & 0.17 \\
\hline Coal & 52.89 & Solar Thermal and Photovoltaic & 0.27 \\
\hline Hydroelectric Conventional & 4.40 & Wood and Wood Derived Fuels & 1.75 \\
\hline Natural Gas & 20.13 & \multicolumn{2}{|l|}{ North Dakota (ND) } \\
\hline Nuclear & 19.81 & Coal & 78.46 \\
\hline Other & 0.03 & Hydroelectric Conventional & 5.29 \\
\hline Other Biomass & 0.17 & Natural Gas & 0.15 \\
\hline Petroleum & 0.07 & Other & 0.11 \\
\hline Wood and Wood Derived Fuels & 2.48 & Other Biomass & 0.02 \\
\hline
\end{tabular}




\begin{tabular}{|c|c|c|c|}
\hline \multicolumn{2}{|l|}{ Arizona (AZ) } & \multirow{2}{*}{$\begin{array}{l}\text { Other Gases } \\
\text { Petroleum }\end{array}$} & \multirow{2}{*}{$\begin{array}{l}0.12 \\
0.09\end{array}$} \\
\hline Coal & 38.39 & & \\
\hline Hydroelectric Conventional & 5.22 & Wind & 15.76 \\
\hline Natural Gas & 26.20 & \multicolumn{2}{|l|}{ Nebraska (NE) } \\
\hline Nuclear & 27.74 & Coal & 72.14 \\
\hline Other Biomass & 0.06 & Hydroelectric Conventional & 3.03 \\
\hline Petroleum & 0.04 & Natural Gas & 1.18 \\
\hline Solar Thermal and Photovoltaic & 1.86 & Nuclear & 18.50 \\
\hline Wind & 0.40 & Other Biomass & 0.18 \\
\hline Wood and Wood Derived Fuels & 0.09 & Petroleum & 0.11 \\
\hline California (CA) & & Wind & 4.86 \\
\hline Coal & 0.44 & \multicolumn{2}{|l|}{ New Hampshire (NH) } \\
\hline Hydroelectric Conventional & 12.66 & Coal & 7.40 \\
\hline Natural Gas & 63.72 & Hydroelectric Conventional & 7.22 \\
\hline Nuclear & 9.55 & Natural Gas & 20.73 \\
\hline Other & 0.44 & Nuclear & 55.25 \\
\hline Other Biomass & 1.52 & Other & 0.31 \\
\hline Other Gases & 0.75 & Other Biomass & 0.69 \\
\hline Petroleum & 0.04 & Petroleum & 0.53 \\
\hline Solar Thermal and Photovoltaic & 2.03 & Wind & 1.97 \\
\hline Wind & 6.84 & Wood and Wood Derived Fuels & 5.91 \\
\hline Wood and Wood Derived Fuels & 2.02 & \multicolumn{2}{|l|}{ New Jersey (NJ) } \\
\hline \multicolumn{2}{|l|}{ Colorado (CO) } & Coal & 3.11 \\
\hline Coal & 63.33 & Hydroelectric Conventional & 0.03 \\
\hline Hydroelectric Conventional & 2.28 & Natural Gas & 41.69 \\
\hline Natural Gas & 20.12 & Nuclear & 51.39 \\
\hline Other & 0.09 & Other & 0.96 \\
\hline Other Biomass & 0.15 & Other Biomass & 1.54 \\
\hline Petroleum & 0.02 & Other Gases & 0.34 \\
\hline Solar Thermal and Photovoltaic & 0.47 & Petroleum & 0.25 \\
\hline Wind & 13.54 & Solar Thermal and Photovoltaic & 0.67 \\
\hline Wood and Wood Derived Fuels & 0.01 & Wind & 0.02 \\
\hline Connecticut (CT) & & \multicolumn{2}{|l|}{ New Mexico (NM) } \\
\hline Coal & 1.91 & Coal & 67.31 \\
\hline Hydroelectric Conventional & 1.13 & Hydroelectric Conventional & 0.26 \\
\hline Natural Gas & 44.31 & Natural Gas & 25.02 \\
\hline Nuclear & 47.96 & Other Biomass & 0.05 \\
\hline Other & 2.00 & Petroleum & 0.16 \\
\hline Other Biomass & 1.82 & Solar Thermal and Photovoltaic & 1.08 \\
\hline Petroleum & 0.86 & Wind & 6.11 \\
\hline Wood and Wood Derived Fuels & 0.01 & \multicolumn{2}{|l|}{ Nevada (NV) } \\
\hline \multicolumn{2}{|l|}{ Delaware (DE) } & Coal & 15.56 \\
\hline Coal & 19.90 & Hydroelectric Conventional & 7.94 \\
\hline Natural Gas & 76.43 & Natural Gas & 73.33 \\
\hline Other Biomass & 0.74 & Other & 0.08 \\
\hline Other Gases & 2.00 & Other Biomass & 0.07 \\
\hline Petroleum & 0.30 & Other Gases & 0.02 \\
\hline Solar Thermal and Photovoltaic & 0.58 & Petroleum & 0.06 \\
\hline Wind & 0.06 & Solar Thermal and Photovoltaic & 2.21 \\
\hline Florida (FL) & & Wind & 0.74 \\
\hline
\end{tabular}




\begin{tabular}{|c|c|c|c|}
\hline Coal & 20.84 & New York (NY) & \\
\hline Hydroelectric Conventional & 0.11 & Coal & 3.44 \\
\hline Natural Gas & 62.49 & Hydroelectric Conventional & 18.29 \\
\hline Nuclear & 11.93 & Natural Gas & 39.80 \\
\hline Other & 1.39 & Nuclear & 32.77 \\
\hline Other Biomass & 1.04 & Other & 0.65 \\
\hline Petroleum & 1.15 & Other Biomass & 1.23 \\
\hline Solar Thermal and Photovoltaic & 0.09 & Petroleum & 0.74 \\
\hline Wood and Wood Derived Fuels & 0.96 & Solar Thermal and Photovoltaic & 0.05 \\
\hline \multicolumn{2}{|l|}{ Georgia (GA) } & Wind & 2.59 \\
\hline Coal & 33.15 & Wood and Wood Derived Fuels & 0.44 \\
\hline Hydroelectric Conventional & 3.06 & \multicolumn{2}{|l|}{ Ohio (OH) } \\
\hline Natural Gas & 33.23 & Coal & 68.88 \\
\hline Nuclear & 27.11 & Hydroelectric Conventional & 0.40 \\
\hline Other & 0.07 & Natural Gas & 15.80 \\
\hline Other Biomass & 0.34 & Nuclear & 11.74 \\
\hline Petroleum & 0.23 & Other & 0.01 \\
\hline Solar Thermal and Photovoltaic & 0.01 & Other Biomass & 0.35 \\
\hline Wood and Wood Derived Fuels & 2.81 & Other Gases & 0.69 \\
\hline \multicolumn{2}{|l|}{ Hawaii $(\mathrm{HI})$} & Petroleum & 1.01 \\
\hline Coal & 14.05 & Solar Thermal and Photovoltaic & 0.03 \\
\hline Hydroelectric Conventional & 0.78 & Wind & 0.83 \\
\hline Other & 3.94 & Wood and Wood Derived Fuels & 0.25 \\
\hline Other Biomass & 3.30 & \multicolumn{2}{|l|}{ Oklahoma (OK) } \\
\hline Other Gases & 0.41 & Coal & 40.68 \\
\hline Petroleum & 72.28 & Hydroelectric Conventional & 2.95 \\
\hline Solar Thermal and Photovoltaic & 0.19 & Natural Gas & 40.75 \\
\hline Wind & 5.04 & Other Biomass & 0.17 \\
\hline \multicolumn{2}{|l|}{ Iowa (IA) } & Petroleum & 0.01 \\
\hline Coal & 58.76 & Wind & 15.14 \\
\hline Hydroelectric Conventional & 1.32 & Wood and Wood Derived Fuels & 0.30 \\
\hline Natural Gas & 2.52 & \multicolumn{2}{|l|}{ Oregon $(\mathrm{OR})$} \\
\hline Nuclear & 9.39 & Coal & 6.29 \\
\hline Other Biomass & 0.28 & Hydroelectric Conventional & 55.41 \\
\hline Petroleum & 0.25 & Natural Gas & 24.05 \\
\hline Wind & 27.47 & Other & 0.06 \\
\hline \multicolumn{2}{|l|}{ Idaho (ID) } & Other Biomass & 0.49 \\
\hline Coal & 0.60 & Petroleum & 0.01 \\
\hline Hydroelectric Conventional & 55.94 & Solar Thermal and Photovoltaic & 0.03 \\
\hline Natural Gas & 22.39 & Wind & 12.48 \\
\hline Other & 0.51 & Wood and Wood Derived Fuels & 1.17 \\
\hline Other Biomass & 1.31 & \multicolumn{2}{|l|}{ Pennsylvania (PA) } \\
\hline Wind & 16.24 & Coal & 38.91 \\
\hline Wood and Wood Derived Fuels & 3.00 & Hydroelectric Conventional & 1.11 \\
\hline \multicolumn{2}{|l|}{ Illinois (IL) } & Natural Gas & 21.97 \\
\hline Coal & 43.31 & Nuclear & 34.63 \\
\hline Hydroelectric Conventional & 0.06 & Other & 0.37 \\
\hline Natural Gas & 3.36 & Other Biomass & 0.81 \\
\hline Nuclear & 47.85 & Other Gases & 0.29 \\
\hline Other & 0.14 & Petroleum & 0.20 \\
\hline
\end{tabular}




\begin{tabular}{|c|c|c|c|}
\hline Other Biomass & 0.30 & Solar Thermal and Photovoltaic & 0.03 \\
\hline Other Gases & 0.18 & Wind & 1.47 \\
\hline Petroleum & 0.04 & Wood and Wood Derived Fuels & 0.22 \\
\hline Solar Thermal and Photovoltaic & 0.03 & \multicolumn{2}{|l|}{ Rhode Island (RI) } \\
\hline Wind & 4.74 & Hydroelectric Conventional & 0.07 \\
\hline \multicolumn{2}{|l|}{ Indiana (IN) } & Natural Gas & 98.28 \\
\hline Coal & 83.94 & Other Biomass & 0.77 \\
\hline Hydroelectric Conventional & 0.35 & Petroleum & 0.81 \\
\hline Natural Gas & 8.18 & Solar Thermal and Photovoltaic & 0.03 \\
\hline Other & 0.40 & Wind & 0.04 \\
\hline Other Biomass & 0.34 & \multicolumn{2}{|l|}{ South Carolina (SC) } \\
\hline Other Gases & 2.18 & Coal & 25.41 \\
\hline Petroleum & 1.42 & Hydroelectric Conventional & 3.29 \\
\hline Solar Thermal and Photovoltaic & 0.03 & Natural Gas & 12.32 \\
\hline Wind & 3.15 & Nuclear & 56.49 \\
\hline \multicolumn{2}{|l|}{ Kansas (KS) } & Other & 0.06 \\
\hline Coal & 61.41 & Other Biomass & 0.22 \\
\hline Hydroelectric Conventional & 0.03 & Petroleum & 0.11 \\
\hline Natural Gas & 4.09 & Solar Thermal and Photovoltaic & 0.00 \\
\hline Nuclear & 14.79 & Wood and Wood Derived Fuels & 2.10 \\
\hline Other Biomass & 0.12 & \multicolumn{2}{|l|}{ South Dakota (SD) } \\
\hline Petroleum & 0.11 & Coal & 28.19 \\
\hline Wind & 19.46 & Hydroelectric Conventional & 40.19 \\
\hline \multicolumn{2}{|l|}{ Kentucky (KY) } & Natural Gas & 4.97 \\
\hline Coal & 92.83 & Petroleum & 0.07 \\
\hline Hydroelectric Conventional & 3.65 & Wind & 26.59 \\
\hline Natural Gas & 1.58 & \multicolumn{2}{|l|}{ Tennessee (TN) } \\
\hline Other & 0.01 & Coal & 40.76 \\
\hline Other Biomass & 0.11 & Hydroelectric Conventional & 15.61 \\
\hline Petroleum & 1.57 & Natural Gas & 6.29 \\
\hline Wood and Wood Derived Fuels & 0.25 & Nuclear & 35.75 \\
\hline \multicolumn{2}{|l|}{ Louisiana (LA) } & Other Biomass & 0.11 \\
\hline Coal & 20.43 & Other Gases & 0.02 \\
\hline Hydroelectric Conventional & 1.02 & Petroleum & 0.16 \\
\hline Natural Gas & 51.48 & Solar Thermal and Photovoltaic & 0.03 \\
\hline Nuclear & 16.62 & Wind & 0.06 \\
\hline Other & 0.67 & Wood and Wood Derived Fuels & 1.20 \\
\hline Other Biomass & 0.08 & \multicolumn{2}{|l|}{ Texas (TX) } \\
\hline Other Gases & 2.20 & Coal & 34.47 \\
\hline Petroleum & 4.85 & Hydroelectric Conventional & 0.11 \\
\hline Wood and Wood Derived Fuels & 2.65 & Natural Gas & 47.03 \\
\hline \multicolumn{2}{|l|}{ Massachusetts (MA) } & Nuclear & 8.84 \\
\hline Coal & 11.91 & Other & 0.06 \\
\hline Hydroelectric Conventional & 2.98 & Other Biomass & 0.17 \\
\hline Natural Gas & 63.92 & Other Gases & 0.55 \\
\hline Nuclear & 13.02 & Petroleum & 0.22 \\
\hline Other & 2.63 & Solar Thermal and Photovoltaic & 0.04 \\
\hline Other Biomass & 3.19 & Wind & 8.28 \\
\hline Petroleum & 1.17 & Wood and Wood Derived Fuels & 0.23 \\
\hline Solar Thermal and Photovoltaic & 0.32 & Utah (UT) & \\
\hline
\end{tabular}




\begin{tabular}{|c|c|c|c|}
\hline & & & \\
\hline Wind & 0.62 & Coal & 81.25 \\
\hline Wood and Wood Derived Fuels & 0.23 & Hydroelectric Conventional & 1.20 \\
\hline Maryland (MD) & & Natural Gas & 15.66 \\
\hline Coal & 43.34 & Other & 0.38 \\
\hline Hydroelectric Conventional & 4.82 & Other Biomass & 0.17 \\
\hline Natural Gas & 8.05 & Petroleum & 0.06 \\
\hline Nuclear & 39.79 & Solar Thermal and Photovoltaic & 0.00 \\
\hline Other & 0.84 & Wind & 1.28 \\
\hline Other Biomass & 1.15 & Virginia (VA) & \\
\hline Petroleum & 0.53 & Coal & 27.10 \\
\hline Solar Thermal and Photovoltaic & 0.18 & Hydroelectric Conventional & 1.61 \\
\hline Wind & 0.90 & Natural Gas & 29.01 \\
\hline Wood and Wood Derived Fuels & 0.40 & Nuclear & 37.56 \\
\hline Maine (ME) & & Other & 0.61 \\
\hline Coal & 0.45 & Other Biomass & 1.23 \\
\hline Hydroelectric Conventional & 25.38 & Petroleum & 0.40 \\
\hline Natural Gas & 34.74 & Wood and Wood Derived Fuels & 2.49 \\
\hline Other & 2.86 & Vermont (VT) & \\
\hline Other Biomass & 1.57 & Hydroelectric Conventional & 18.68 \\
\hline Petroleum & 1.70 & Natural Gas & 0.04 \\
\hline Wind & 7.47 & Nuclear & 70.39 \\
\hline Wood and Wood Derived Fuels & 25.84 & Other Biomass & 0.37 \\
\hline Michigan (MI) & & Petroleum & 0.07 \\
\hline Coal & 52.96 & Solar Thermal and Photovoltaic & 0.25 \\
\hline Hydroelectric Conventional & 1.34 & Wind & 3.43 \\
\hline Natural Gas & 11.61 & Wood and Wood Derived Fuels & 6.76 \\
\hline Nuclear & 27.21 & Washington (WA) & \\
\hline Other & 0.29 & Coal & 5.90 \\
\hline Other Biomass & 0.93 & Hydroelectric Conventional & 68.46 \\
\hline Other Gases & 0.90 & Natural Gas & 10.01 \\
\hline Petroleum & 0.50 & Nuclear & 7.41 \\
\hline Wind & 2.63 & Other & 0.11 \\
\hline Wood and Wood Derived Fuels & 1.62 & Other Biomass & 0.25 \\
\hline Minnesota (MN) & & Other Gases & 0.36 \\
\hline Coal & 45.85 & Petroleum & 0.02 \\
\hline Hydroelectric Conventional & 1.00 & Solar Thermal and Photovoltaic & 0.00 \\
\hline Natural Gas & 12.28 & Wind & 6.14 \\
\hline Nuclear & 20.87 & Wood and Wood Derived Fuels & 1.34 \\
\hline Other & 0.70 & Wisconsin (WI) & \\
\hline Other Biomass & 1.12 & Coal & 61.62 \\
\hline Petroleum & 0.05 & Hydroelectric Conventional & 3.00 \\
\hline Solar Thermal and Photovoltaic & 0.01 & Natural Gas & 12.28 \\
\hline Wind & 16.10 & Nuclear & 17.70 \\
\hline Wood and Wood Derived Fuels & 2.02 & Other & 0.10 \\
\hline Missouri (MO) & & Other Biomass & 0.73 \\
\hline Coal & 83.33 & Petroleum & 0.46 \\
\hline Hydroelectric Conventional & 1.24 & Wind & 2.36 \\
\hline Natural Gas & 4.82 & Wood and Wood Derived Fuels & 1.74 \\
\hline Nuclear & 9.16 & West Virginia (WV) & \\
\hline Other & 0.02 & Coal & 95.28 \\
\hline
\end{tabular}




\begin{tabular}{lclc} 
Other Biomass & 0.07 & Hydroelectric Conventional & 2.29 \\
Petroleum & 0.07 & Natural Gas & 0.36 \\
Wind & 1.28 & Other Biomass & 0.01 \\
Wood and Wood Derived Fuels & 0.01 & Other Gases & 0.04 \\
\cline { 1 - 1 } Mississippi (MS) & & Petroleum & 0.20 \\
\cline { 1 - 2 } Coal & 16.48 & Wind & 1.83 \\
\cline { 2 - 3 } Natural Gas & 60.17 & Wyoming (WY) & 88.48 \\
Nuclear & 20.57 & Coal & 1.35 \\
Other & 0.01 & Hydroelectric Conventional & 0.98 \\
Other Biomass & 0.03 & Natural Gas & 0.13 \\
Petroleum & 0.03 & Other & 0.54 \\
Wood and Wood Derived Fuels & 2.71 & Other Gases & 0.07 \\
& & Petroleum & 8.45 \\
\hline
\end{tabular}

\section{Life cycle GHG emissions intensity for different electricity generation technologies}

Table S6 Life cycle GHG emissions intensity for different electricity generation technologies

\begin{tabular}{lcc}
\hline Fuel Source & Database & $\begin{array}{c}\text { GHG emission factor for } \\
\text { producing 1 MJ electricity }\end{array}$ \\
\hline Coal & USLCI $^{9}$ & 0.301 \\
Hydroelectric Conventional & ELCD $^{10}$ & 0.00678 \\
Natural gas & USLCI $^{9}$ & 0.203 \\
Nuclear & USLCI $^{9}$ & 0.00322 \\
Other & Ecoinvent $^{11}$ & 0.0407 \\
Other Biomass & USLCI $^{9}$ & 0.0126 \\
Other Gases & Ecoinvent $^{11}$ & 0.524 \\
Petroleum & USLCI $^{9}$ & 0.315 \\
Solar Thermal and Photovoltaic & Ecoinvent $^{11}$ & 0.0132 \\
Wind & ELCD $^{10}$ & 0.00184 \\
Wood and Wood Derived Fuels & USLCI $^{9}$ & 0.0144 \\
\hline
\end{tabular}

\section{Exergy content of available waste heat}

The waste heat estimation procedure described in the methodology section of main paper only takes into account the first law of thermodynamics and quantifies the thermal energy content of waste heat streams available at existing NG CS. The first law of thermodynamics has the limitation of assuming substitutability between energy resources and failing to distinguish between the quality of energy (e.g. heat vs work). For example, as per the first law of thermodynamics, 1 Joule of heat available at $400^{\circ} \mathrm{K}$ is substitutable with 1 Joule of heat at $500^{\circ} \mathrm{K}$. We address this limitation by quantifying the exergy content of available waste heat at existing NG CS. Exergy represents the maximum amount of work that can be extracted from a 
system when it is brought in thermodynamic equilibrium with the surroundings (also defined as the reference state). Exergy is particularly appealing since it considers both the first and second law of thermodynamics and provides a better representation of the ability of different energy streams to do work. ${ }^{12}$ Specifically, we quantify the thermal aspect of exergy, ${ }^{13} \mathrm{E}$, which is induced by the temperature difference between exhaust stream and the surrounding environment using eq $7^{14}$ where $\mathrm{Q}$ and $\mathrm{T}$ are the energy and temperature of exhaust stream, and $\mathrm{T}_{0}$ is the ambient temperature. The results for exergy content of available waste heat from NG CS are provided Figure S3.

$E=Q \cdot\left|1-\frac{T_{0}}{T-T_{0}} \ln \frac{T}{T_{0}}\right|$

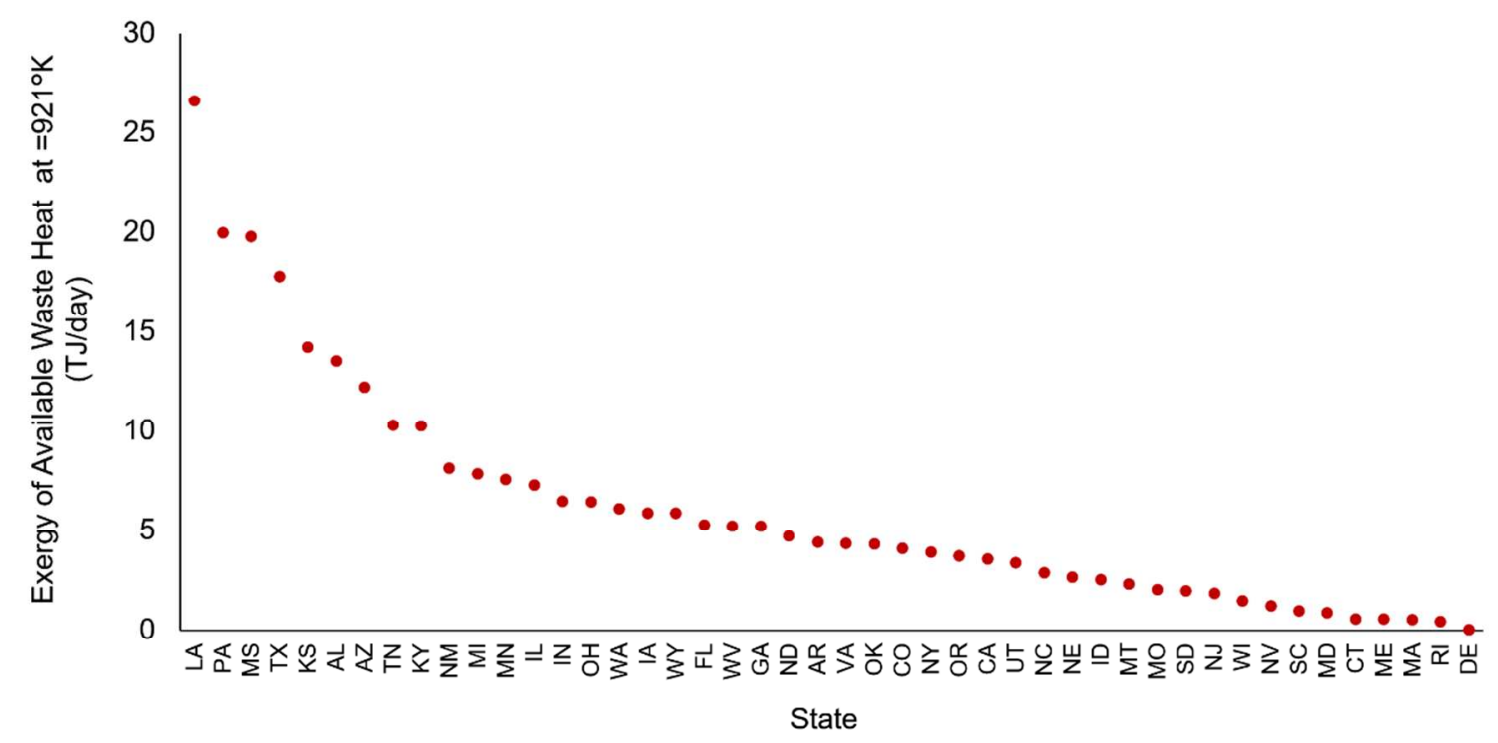

Figure S3 Exergy content of available waste heat at NG CS by state 


\section{References}

1. STI (Strive To Innovate) group. http://setxind.com/midstream/compressor-stations-whathow-why/ 2015.

2. Poinsot, T.; Veynante, D., Theoretical and Numerical Combustion, 2011. URL http://elearning. cerfacs. fr/combustion/onlinePoinsotBook/onlinethirdedition/index. php 2014.

3. (a) Kuo, K. K., Principles of combustion. 1986; (b) Williams, G., Combustion theory. 1985.

4. Goodwin, D.; Malaya, N.; Moffat, H.; Speth, R., Cantera: An object-oriented software toolkit for chemical kinetics, thermodynamics, and transport processes. Caltech, Pasadena, CA 2009.

5. Johnson, I.; William, T.; Choate, W.; Amber Davidson, A., Waste heat recovery: technology and opportunities in US industry. US Department of Energy, Office of Energy Efficiency and Renewable Energy, Industrial Technologies Program 2008.

6. Moran, M. J.; Shapiro, H. N.; Boettner, D. D.; Bailey, M. B., Fundamentals of engineering thermodynamics. John Wiley \& Sons: 2010.

7. Braun, R.; Klein, S.; Reindl, D., Evaluation of system configurations for solid oxide fuel cell-based micro-combined heat and power generators in residential applications. Journal of Power Sources 2006, 158 (2), 1290-1305.

8. EIA, Energy Information Administration, http://www.eia.gov/electricity/data/state/. 2013.

9. USLCI, U.S. Life Cycle Inventory Database. National Renewable Energy Laboratory

\section{2.}

10. ELCD, European Commission, Joint Research Centre - Institute for Environment and Sustainability and DG Environment - Directorate G. European Reference Life Cycle Database, version 2.02008.

11. Weidema, B.; Bauer, C.; Hischier, R.; Mutel, C.; Nemecek, T.; Reinhard, J.; Vadenbo, C.; Wernet, G., The Ecoinvent Database: Overview and Methodology, Data Quality Guideline for the Ecoinvent Database Version 3. 2013.

12. (a) Szargut, J.; Morris, D. R.; Steward, F. R., Exergy analysis of thermal, chemical, and metallurgical processes. 1987; (b) Szargut, J., Exergy method: technical and ecological applications. WIT press: 2005; Vol. 18; (c) Dewulf, J.; Van Langenhove, H.; Muys, B.; Bruers, S.; Bakshi, B. R.; Grubb, G. F.; Paulus, D.; Sciubba, E., Exergy: its potential and limitations in environmental science and technology. Environmental Science \& Technology 2008, 42 (7), 2221-2232.

13. Palazzo, P., Thermal and mechanical aspect of entropy-exergy relationship. International Journal of Energy and Environmental Engineering 2012, 3 (1), 1-10.

14. Wall, G., Exergetics. 2009. 